\title{
The Approximate Solution of Some Plane Boundary Value Problems of the Moment Theory of Elasticity
}

\author{
Roman Janjgava \\ Ilia Vekua Institute of Applied Mathematics, Ivane Javakhishvili Tbilisi State University, University Street 2, 0186 Tbilisi, Georgia \\ Correspondence should be addressed to Roman Janjgava; roman.janjgava@gmail.com
}

Received 14 August 2015; Revised 19 January 2016; Accepted 31 January 2016

Academic Editor: Mikhail Panfilov

Copyright (C) 2016 Roman Janjgava. This is an open access article distributed under the Creative Commons Attribution License, which permits unrestricted use, distribution, and reproduction in any medium, provided the original work is properly cited.

\begin{abstract}
We consider a two-dimensional system of differential equations of the moment theory of elasticity. The general solution of this system is represented by two arbitrary harmonic functions and solution of the Helmholtz equation. Based on the general solution, an algorithm of constructing approximate solutions of boundary value problems is developed. Using the proposed method, the approximate solutions of some problems on stress concentration on the contours of holes are constructed. The values of stress concentration coefficients obtained in the case of moment elasticity and for the classical elastic medium are compared. In the final part of the paper, we construct the approximate solution of a nonlocal problem whose exact solution is already known and compare our approximate solution with the exact one. Supposedly, the proposed method makes it possible to construct approximate solutions of quite a wide class of boundary value problems.
\end{abstract}

\section{Introduction}

An elastic medium which at every point is characterized not only by the displacement vector but also by the rotation vector is called the Cosserat medium after the Cosserat brothers who considered the deformation of such a medium for the first time as far back as 1909 [1]. The corresponding theory is often called the moment or asymmetrical theory of elasticity because its deformation and stress tensors are not symmetrical. The development of this theory along various lines attained its peak in the sixties-seventies of the last century [2-12]. Though, in subsequent years, more general models of a micropolar elastic medium were proposed and studied [13-15], the investigation of problems related to the Cosserat theory is still going on [16-36]. The popularity of the theory can possibly be explained by its physical clarity and a relative mathematical simplicity.

In the present paper, we consider the static case of plane deformation of the elastic Cosserat medium. The corresponding basic equilibrium equations are given in Section 2. For constructing approximate solutions of boundary value problems, a semianalytical method is proposed, which is based on using the representation of the general solution by solutions of simpler equations of mathematical physics.
In Section 3 we construct the general solution of the corresponding system of equations using two harmonic functions and a solution of the Helmholtz equation. As is known, the method of representation of the general solution by the well-studied functions of mathematical physics is widely applied to the construction of analytical (exact) solutions of boundary value problems, but one succeeds in obtaining analytical solutions of boundary value problems only for some particular types of boundary conditions when the considered domain has a regular configuration.

Section 4 deals with the application of the obtained general solution to the construction of approximate solutions of boundary value problems, which allows us to consider a wide class of boundary value problems without imposing special restrictions on the domain geometry. When the functions appearing in the general solution are expanded into series in Green's functions and the boundary conditions are satisfied at the points of the domain boundary, we obtain a system of linear algebraic equations for the unknown coefficients of the series. After solving this system and again using the general representation of the solution, we can write an analytical approximate solution which satisfies the basic system of equations up to the domain boundary. The described algorithm is similar to the method of fundamental 
solutions [37-39] and the boundary elements method [4044]. However it is simpler than the method of fundamental solutions for which the integral equation is considered on some contour that encircles the domain [39]. As different from the boundary elements method, the boundary does not have to be approximated by boundary elements and there is no need to use local coordinate systems [40], which also simplifies the construction of an approximate solution. But the main difference of our approach from the abovementioned two methods is that in our case it becomes possible not to use Green's functions at all. At the end of Section 4, the harmonic functions and the Helmholtz function in the general solution are represented as finite series obtained as a result of the separation of variables in a polar system of coordinates. Further the Cartesian coordinates of the points on the boundary are substituted in the representations of the functions whose values are given on the boundary and the obtained expressions are equated to the respective values of the boundary conditions at the same points. As a result we obtain a system of linear algebraic equations and after solving it we can easily write an approximate solution of the considered boundary value problem.

In Section 5, we construct by the proposed method the approximate solutions of the boundary value problems of stress concentration in the rectangular domains with circular holes. The coefficients of stress concentration along the hole contour are calculated and we compare the stress concentration values obtained in the case of moment elasticity and for the classical elastic medium. For the classical elastic medium we also construct the approximate solution of the nonlocal problem with the known exact solution and compare our approximate solution with the exact one.

\section{Basic Relations of the Plane Moment Theory of Elasticity}

Let $O x y z$ be the Cartesian coordinate system with unit vectors $\mathbf{i}, \mathbf{j}, \mathbf{k}$. Consider the plane deformed state of the moment-elastic medium which is parallel to the $O x y$-plane. In that case, the displacement vector $\mathbf{u}$ and the rotation vector $\mathbf{w}$ at the points of the medium have the form

$$
\begin{aligned}
\mathbf{u} & =u(x, y) \mathbf{i}+v(x, y) \mathbf{j}, \\
\mathbf{w} & =\omega(x, y) \mathbf{k} .
\end{aligned}
$$

Homogeneous equations of static equilibrium in terms of displacement and rotation components are written as [10]

$$
\begin{array}{r}
(\mu+\alpha) \Delta u+(\lambda+\mu-\alpha) \partial_{x}\left(\partial_{x} u+\partial_{y} v\right)+2 \alpha \partial_{y} \omega=0 \\
(\mu+\alpha) \Delta v+(\lambda+\mu-\alpha) \partial_{y}\left(\partial_{x} u+\partial_{y} v\right)-2 \alpha \partial_{x} \omega=0 \\
(\nu+\beta) \Delta \omega+2 \alpha\left(\partial_{x} v-\partial_{y} u\right)-4 \alpha \omega=0
\end{array}
$$

where $\lambda, \mu$ are the Lamé constants; $\alpha, \beta, \nu$ are the constants characterizing the microstructure of the considered medium; and $\partial_{x} \equiv \partial / \partial x, \partial_{y} \equiv \partial / \partial y$, and $\Delta \equiv \partial_{x x}+\partial_{y y}$.
Relations of Hooke's law for the moment-elastic medium have the form

$$
\begin{aligned}
& \sigma_{x x}=\lambda\left(\partial_{x} u+\partial_{y} v\right)+2 \mu \partial_{x} u, \\
& \sigma_{y y}=\lambda\left(\partial_{x} u+\partial_{y} v\right)+2 \mu \partial_{y} v, \\
& \sigma_{x y}=(\mu+\alpha) \partial_{x} v+(\mu-\alpha) \partial_{y} u-2 \alpha \omega, \\
& \sigma_{y x}=(\mu+\alpha) \partial_{y} u+(\mu-\alpha) \partial_{x} v+2 \alpha \omega, \\
& \sigma_{z z}=\lambda\left(\partial_{x} u+\partial_{y} v\right), \\
& \mu_{x z}=(\nu+\beta) \partial_{x} \omega, \\
& \mu_{y z}=(\nu+\beta) \partial_{y} \omega, \\
& \mu_{z x}=(\nu-\beta) \partial_{x} \omega, \\
& \mu_{z y}=(\nu-\beta) \partial_{y} \omega,
\end{aligned}
$$

where $\sigma_{x x}, \ldots, \sigma_{y x}$ are the stress tensor components and $\mu_{x z}, \ldots, \mu_{z y}$ are the moment stress tensor components. All other stress tensor and moment stress tensor components are assumed in the case of plane deformation to be equal to zero.

\section{The General Solution of System (2)}

The general solution of system (2) can be represented by two arbitrary harmonic functions and an arbitrary solution of the Helmholtz equation.

We introduce the following notation:

$$
\begin{aligned}
& \theta=(\lambda+2 \mu)\left(\partial_{x} u+\partial_{y} v\right), \\
& \eta=(\mu+\alpha)\left(\partial_{x} v-\partial_{y} u\right)-2 \alpha \omega .
\end{aligned}
$$

Using (4), the first two equations of system (2) can be written as follows:

$$
\begin{aligned}
& \partial_{x} \theta-\partial_{y} \eta=0, \\
& \partial_{y} \theta+\partial_{x} \eta=0 .
\end{aligned}
$$

From system (5) we see that $\theta$ and $\eta$ are the self-conjugate harmonic functions

$$
\begin{aligned}
& \Delta \theta=0, \\
& \Delta \eta=0
\end{aligned}
$$

and represent them in the form

$$
\begin{aligned}
& \theta=a\left(\partial_{x} \varphi+\partial_{y} \psi\right), \\
& \eta=a\left(\partial_{x} \psi-\partial_{y} \varphi\right),
\end{aligned}
$$

where $\varphi$ and $\psi$ are arbitrary harmonic functions and $a$ is any nonzero real constant.

Remark. In representations (7) only one harmonic function could have been used, but we have introduced two harmonic 
functions to obtain the general solution which is more convenient for our purpose.

With (7) taken into consideration, from relations (4) we have

$$
\begin{aligned}
& \partial_{x} u+\partial_{y} v=\frac{a}{\lambda+2 \mu}\left(\partial_{x} \varphi+\partial_{y} \psi\right), \\
& \partial_{x} v-\partial_{y} u=\frac{a}{\mu+\alpha}\left(\partial_{x} \psi-\partial_{y} \varphi\right)+\frac{2 \alpha}{\mu+\alpha} \omega .
\end{aligned}
$$

Let us substitute the second formula (8) in the third equation of system (2). After simple transformations we obtain the equation

$$
\begin{aligned}
\Delta \omega & -\frac{4 \mu \alpha}{(\nu+\beta)(\mu+\alpha)} \omega \\
& =-\frac{2 \alpha a}{(\nu+\beta)(\mu+\alpha)}\left(\partial_{x} \psi-\partial_{y} \varphi\right) .
\end{aligned}
$$

In view of the fact that $\varphi$ is a harmonic function, the general solution of (9) can be written in the form

$$
\omega=b \chi+\frac{a}{2 \mu}\left(\partial_{x} \psi-\partial_{y} \varphi\right),
$$

where $\chi$ is an arbitrary solution of the Helmholtz equation

$$
\Delta \chi-\frac{4 \mu \alpha}{(\nu+\beta)(\mu+\alpha)} \chi=0
$$

and $b$ is any nonzero real constant.

Using representation (10) and keeping in mind that $\chi$ is the solution of (11), we write system (8) as follows:

$$
\begin{aligned}
\partial_{x} u+\partial_{y} v= & \frac{a}{\lambda+2 \mu}\left(\partial_{x} \varphi+\partial_{y} \psi\right), \\
\partial_{x} v-\partial_{y} u= & \frac{a}{\mu}\left(\partial_{x} \psi-\partial_{y} \varphi\right) \\
& +\frac{(\nu+\beta) b}{2 \mu}\left(\partial_{x x} \chi+\partial_{y y} \chi\right) .
\end{aligned}
$$

The second equation (12) is satisfied identically if we take

$$
\begin{aligned}
& u=\partial_{x} \Phi+\frac{a}{\mu} \varphi-\frac{(\nu+\beta) b}{2 \mu} \partial_{y} \chi \\
& v=\partial_{y} \Phi+\frac{a}{\mu} \psi+\frac{(\nu+\beta) b}{2 \mu} \partial_{x} \chi .
\end{aligned}
$$

By substituting formulas (13) in the first equation of system (12), we obtain the Poisson equation satisfied by function $\Phi$ :

$$
\Delta \Phi=-\frac{(\lambda+\mu) a}{\mu(\lambda+2 \mu)}\left(\partial_{x} \varphi+\partial_{y} \psi\right) .
$$

Since $\varphi$ and $\psi$ are harmonic functions, we can easily write the general solution of the latter equation:

$$
\begin{aligned}
\Phi= & -\frac{(\lambda+\mu) a}{2 \mu(\lambda+2 \mu)}\left(x\left(\varphi+\partial_{x} \Psi\right)+y\left(\psi+\partial_{y} \Psi\right)\right) \\
& +\frac{a}{\mu} \Psi
\end{aligned}
$$

where $\Psi$ is an arbitrary harmonic function.
The substitution of expression (15) in formula (13) gives

$$
\begin{aligned}
2 \mu u= & \frac{(\lambda+3 \mu) a}{\lambda+2 \mu} \varphi^{*}-\frac{(\lambda+\mu) a}{\lambda+2 \mu}\left(x \partial_{x} \varphi^{*}+y \partial_{x} \psi^{*}\right) \\
& -(\nu+\beta) b \partial_{y} \chi, \\
2 \mu \nu= & \frac{(\lambda+3 \mu) a}{\lambda+2 \mu} \psi^{*}-\frac{(\lambda+\mu) a}{\lambda+2 \mu}\left(x \partial_{y} \varphi^{*}+y \partial_{y} \psi^{*}\right) \\
& +(\nu+\beta) b \partial_{x} \chi,
\end{aligned}
$$

where

$$
\begin{aligned}
& \varphi^{*}=\varphi+\partial_{x} \Psi, \\
& \psi^{*}=\psi+\partial_{y} \Psi .
\end{aligned}
$$

Obviously, $\varphi^{*}$ and $\psi^{*}$ are arbitrary harmonic functions. For simplicity, we denote them by $\varphi$ and $\psi$.

It is convenient to fix arbitrary constants $a$ and $b$ as follows:

$$
\begin{aligned}
& a=\frac{\lambda+2 \mu}{\lambda+\mu}, \\
& b=\frac{1}{\nu+\beta} .
\end{aligned}
$$

Then formulas (16) and (10) take the form

$$
\begin{aligned}
& 2 \mu u=\frac{\lambda+3 \mu}{\lambda+\mu} \varphi-x \partial_{x} \varphi-y \partial_{x} \psi-\partial_{y} \chi \\
& 2 \mu \nu=\frac{\lambda+3 \mu}{\lambda+\mu} \psi-y \partial_{y} \psi-x \partial_{y} \varphi+\partial_{x} \chi \\
& 2 \mu \omega=\frac{2 \mu}{\nu+\beta} \chi+\frac{\lambda+2 \mu}{\lambda+\mu}\left(\partial_{x} \psi-\partial_{y} \varphi\right) .
\end{aligned}
$$

System (19) is the representation of the general solution of system (2) by two arbitrary harmonic functions $\varphi(x, y)$ and $\psi(x, y)$ and an arbitrary solution $\chi(x, y)$ of the Helmholtz equation.

Let us now express stresses and moment stresses through functions $\varphi, \psi$, and $\chi$. To this end, formulas (19) are substituted in relations (3) to obtain

$$
\begin{aligned}
\sigma_{x x}= & \left(\frac{\lambda+2 \mu}{\lambda+\mu} \partial_{x}-x \partial_{x x}\right) \varphi+\left(\frac{\lambda}{\lambda+\mu} \partial_{y}-y \partial_{x x}\right) \psi \\
& -\partial_{x y} \chi \\
\sigma_{y y}= & \left(\frac{\lambda}{\lambda+\mu} \partial_{x}-x \partial_{y y}\right) \varphi+\left(\frac{\lambda+2 \mu}{\lambda+\mu} \partial_{y}-y \partial_{y y}\right) \psi \\
& +\partial_{x y} \chi \\
\sigma_{x y}= & \left(\frac{\mu}{\lambda+\mu} \partial_{y}-x \partial_{x y}\right) \varphi+\left(\frac{\mu}{\lambda+\mu} \partial_{x}-y \partial_{x y}\right) \psi \\
& -\partial_{y y} \chi,
\end{aligned}
$$




$$
\begin{aligned}
\sigma_{y x}= & \left(\frac{\mu}{\lambda+\mu} \partial_{y}-x \partial_{x y}\right) \varphi+\left(\frac{\mu}{\lambda+\mu} \partial_{x}-y \partial_{x y}\right) \psi \\
& +\partial_{x x} \chi, \\
\mu_{x z}= & \frac{(\nu+\beta)(\lambda+2 \mu)}{2 \mu(\lambda+\mu)}\left(\partial_{x x} \psi-\partial_{x y} \varphi\right)+\partial_{x} \chi, \\
\mu_{y z}= & \frac{(\nu+\beta)(\lambda+2 \mu)}{2 \mu(\lambda+\mu)}\left(\partial_{x y} \psi-\partial_{y y} \varphi\right)+\partial_{y} \chi, \\
\mu_{z x}= & \frac{(\nu-\beta)(\lambda+2 \mu)}{2 \mu(\lambda+\mu)}\left(\partial_{x x} \psi-\partial_{x y} \varphi\right)+\frac{\nu-\beta}{\nu+\beta} \partial_{x} \chi, \\
& \frac{(\nu-\beta)(\lambda+2 \mu)}{2 \mu(\lambda+\mu)}\left(\partial_{x y} \psi-\partial_{y y} \varphi\right)+\frac{\nu-\beta}{\nu+\beta} \partial_{y} \chi .
\end{aligned}
$$

Let us assume that $\mathbf{l}$ and $\mathbf{s}$ are mutually normal unit vectors such that

$$
\mathbf{l} \times \mathbf{s}=\mathbf{k} \text {, }
$$

and $\vartheta$ is the angle between vector 1 and the positive direction of the $O x$-axis. Then, by virtue of the transformation formulas of first- and second-rank tensor components, the following formulas hold true:

$$
\begin{aligned}
& u_{l}=u \cos \vartheta+v \sin \vartheta \\
& u_{s}=-u \sin \vartheta+v \cos \vartheta \\
& \sigma_{l l}=\sigma_{x x} \cos ^{2} \vartheta+\left(\sigma_{x y}+\sigma_{y x}\right) \sin \vartheta \cos \vartheta+\sigma_{y y} \sin ^{2} \vartheta \\
& \sigma_{s s}=\sigma_{x x} \sin ^{2} \vartheta-\left(\sigma_{x y}+\sigma_{y x}\right) \sin \vartheta \cos \vartheta+\sigma_{y y} \cos ^{2} \vartheta \\
& \sigma_{l s}=\left(\sigma_{y y}-\sigma_{x x}\right) \sin \vartheta \cos \vartheta+\sigma_{x y} \cos ^{2} \vartheta-\sigma_{y x} \sin ^{2} \vartheta \\
& \sigma_{s l}=\left(\sigma_{y y}-\sigma_{x x}\right) \sin \vartheta \cos \vartheta-\sigma_{x y} \sin ^{2} \vartheta+\sigma_{y x} \cos ^{2} \vartheta \\
& \mu_{l z}=\mu_{x z} \cos \vartheta+\mu_{y z} \sin \vartheta \\
& \mu_{s z}=-\mu_{x z} \sin \vartheta+\mu_{y z} \cos \vartheta \\
& \mu_{z l}=\mu_{z x} \cos \vartheta+\mu_{z y} \sin \vartheta \\
& \mu_{z s}=-\mu_{z x} \sin \vartheta+\mu_{z y} \cos \vartheta .
\end{aligned}
$$

Substituting formulas (19) and (20) in relations (22) we can express normal and tangent displacements as well as stresses and moment stresses acting on the area element of arbitrary orientation through functions $\varphi, \psi$, and $\chi$.

\section{Use of the General Solution for the Construction of Approximate Solutions of Boundary Value Problems}

In this section it is shown how the general solution derived in the preceding section can be used for constructing approximate solutions of boundary value problems. The technique to be described here may be called a semianalytical method in view of the fact that approximate solutions are written in analytical form. In this sense, it is analogous to the method of fundamental solutions [37-39] and the boundary elements method [40-44], but there are differences which have been mentioned in Introduction.

So, we start the description of the algorithm of constructing the approximate solution of a boundary value problem when Green's functions are used.

In the general solutions (19) and (20), the harmonic functions $\varphi_{j}$ and $\psi_{j}$ are taken for each index $j=1,2, \ldots, N$ ( $N$ is some natural number) in the following manner:

$$
\left(\varphi_{j}, \psi_{j}\right)=\left(a_{j}, b_{j}\right) \ln \sqrt{x^{2}+y^{2}}
$$

and solution $\chi_{j}$ of the Helmholtz equation is represented as

$$
\chi_{j}=c_{j} K_{0}\left(\gamma \sqrt{x^{2}+y^{2}}\right)
$$

where $K_{0}\left(\gamma \sqrt{x^{2}+y^{2}}\right)$ is a modified Bessel function of second kind or a Macdonald function (of zeroth order) which tends to infinity at point $(0,0)$ and vanishes at infinity [45] and $\gamma=\sqrt{4 \mu \alpha /(\nu+\beta)(\mu+\alpha)}$ and $a_{j}, b_{j}, c_{j}, j=1,2, \ldots, N$, are the real coefficients to be defined.

By formula (23) the first-order partial derivatives of the harmonic functions $\varphi_{j}$ and $\psi_{j}$ have the form

$$
\begin{aligned}
& \left(\partial_{x} \varphi_{j}, \partial_{x} \psi_{j}\right)=\left(a_{j}, b_{j}\right) \frac{x}{x^{2}+y^{2}}, \\
& \left(\partial_{y} \varphi_{j}, \partial_{y} \psi_{j}\right)=\left(a_{j}, b_{j}\right) \frac{y}{x^{2}+y^{2}},
\end{aligned}
$$

and the partial derivatives of the metaharmonic functions $\chi_{j}$ are written as

$$
\begin{aligned}
& \partial_{x} \chi_{j}=-c_{j} \frac{\gamma x K_{1}\left(\gamma \sqrt{x^{2}+y^{2}}\right)}{\sqrt{x^{2}+y^{2}}}, \\
& \partial_{y} \chi_{j}=-c_{j} \frac{\gamma y K_{1}\left(\gamma \sqrt{x^{2}+y^{2}}\right)}{\sqrt{x^{2}+y^{2}}} .
\end{aligned}
$$

The second derivatives of these functions appear in formulas (20):

$$
\begin{gathered}
\left(\partial_{x x} \varphi_{j}, \partial_{x x} \psi_{j}\right)=-\left(\partial_{y y} \varphi_{j}, \partial_{y y} \psi_{j}\right)=\left(a_{j}, b_{j}\right) \\
\cdot \frac{y^{2}-x^{2}}{\left(x^{2}+y^{2}\right)^{2}} \\
\left(\partial_{x y} \varphi_{j}, \partial_{x y} \psi_{j}\right)=-\left(a_{j}, b_{j}\right) \frac{2 x y}{\left(x^{2}+y^{2}\right)^{2}}
\end{gathered}
$$




$$
\begin{gathered}
\partial_{x x} \chi_{j}=c_{j} \frac{\gamma^{2}}{2}\left(K_{0}\left(\gamma \sqrt{x^{2}+y^{2}}\right)\right. \\
\left.-\frac{y^{2}-x^{2}}{x^{2}+y^{2}} K_{2}\left(\gamma \sqrt{x^{2}+y^{2}}\right)\right), \\
\partial_{y y} \chi_{j}=c_{j} \frac{\gamma^{2}}{2}\left(K_{0}\left(\gamma \sqrt{x^{2}+y^{2}}\right)\right. \\
\left.+\frac{y^{2}-x^{2}}{x^{2}+y^{2}} K_{2}\left(\gamma \sqrt{x^{2}+y^{2}}\right)\right), \\
\partial_{x y} \chi_{j}=\sum_{j=1}^{N} c_{j} \frac{\gamma^{2} x y}{x^{2}+y^{2}} K_{2}\left(\gamma \sqrt{x^{2}+y^{2}}\right) .
\end{gathered}
$$

If we substitute the corresponding expressions (23)-(26) in formulas (19), then for each index $j=1,2, \ldots, N$ we obtain solution $\left(2 \mu u_{j}, 2 \mu v_{j}, 2 \mu \omega_{j}\right)$ of system (2) satisfying this system everywhere except the origin where it has a singularity:

$$
\begin{aligned}
2 \mu u_{j}(x, y)= & \left(\frac{\lambda+3 \mu}{\lambda+\mu} \ln \sqrt{x^{2}+y^{2}}-\frac{x^{2}}{x^{2}+y^{2}}\right) a_{j} \\
& -\frac{x y}{x^{2}+y^{2}} b_{j} \\
& +\frac{y K_{1}\left(\gamma \sqrt{x^{2}+y^{2}}\right)}{\sqrt{x^{2}+y^{2}}} c_{j}, \\
2 \mu v_{j}(x, y)= & -\frac{x y}{x^{2}+y^{2}} a_{j} \\
& +\left(\frac{\lambda+3 \mu}{\lambda+\mu} \ln \sqrt{x^{2}+y^{2}}-\frac{y^{2}}{x^{2}+y^{2}}\right) b_{j} \\
& -\frac{x K_{1}\left(\gamma \sqrt{x^{2}+y^{2}}\right)}{\sqrt{x^{2}+y^{2}}} c_{j}, \\
& +\frac{2 \mu}{v+\beta} K_{0}\left(\gamma \sqrt{x^{2}+y^{2}}\right) c_{j} . \\
2 \mu \omega_{j}(x, y)= & \frac{\lambda+2 \mu}{\lambda+\mu} \frac{1}{x^{2}+y^{2}}\left(-y a_{j}+x b_{j}\right) \\
& \left.+\frac{2}{2}\right)
\end{aligned}
$$

The values of stresses and moment stresses $\sigma_{x x}, \sigma_{y y}, \ldots, \mu_{y z}$ are obtained if representations (25)-(27) are substituted in the respective formulas (20):

$$
\begin{aligned}
& \sigma_{x x j}(x, y)=\left(\frac{\lambda+2 \mu}{\lambda+\mu} \frac{x}{x^{2}+y^{2}}-\frac{x\left(y^{2}-x^{2}\right)}{\left(x^{2}+y^{2}\right)^{2}}\right) a_{j} \\
& -\left(\frac{\lambda}{\lambda+\mu} \frac{y}{x^{2}+y^{2}}-\frac{y\left(y^{2}-x^{2}\right)}{\left(x^{2}+y^{2}\right)^{2}}\right) b_{j}-\frac{\gamma^{2} x y}{x^{2}+y^{2}} \\
& \cdot K_{2}\left(\gamma \sqrt{x^{2}+y^{2}}\right) c_{j},
\end{aligned}
$$

$$
\begin{gathered}
\sigma_{y y j}(x, y)=\left(\frac{\lambda}{\lambda+\mu} \frac{x}{x^{2}+y^{2}}+\frac{x\left(y^{2}-x^{2}\right)}{\left(x^{2}+y^{2}\right)^{2}}\right) a_{j} \\
+\left(\frac{\lambda+2 \mu}{\lambda+\mu} \frac{y}{x^{2}+y^{2}}+\frac{y\left(y^{2}-x^{2}\right)}{\left(x^{2}+y^{2}\right)^{2}}\right) b_{j}+\frac{\gamma^{2} x y}{x^{2}+y^{2}} \\
\cdot K_{2}\left(\gamma \sqrt{x^{2}+y^{2}}\right) c_{j}, \\
\mu_{z y j}(x, y)=\frac{(\nu-\beta)(\lambda+2 \mu)}{2 \mu(\lambda+\mu)}\left(\frac{y^{2}-x^{2}}{\left(x^{2}+y^{2}\right)^{2}} a_{j}\right. \\
\left.-\frac{2 x y}{\left(x^{2}+y^{2}\right)^{2}} b_{j}\right)-\frac{\nu-\beta}{\nu+\beta} \frac{\gamma y K_{1}\left(\gamma \sqrt{x^{2}+y^{2}}\right)}{\sqrt{x^{2}+y^{2}}} c_{j} .
\end{gathered}
$$

After that, each jth function of (28) and (29) is shifted by value $\left(\xi_{j}, \eta_{j}\right)$ (Figure 1). For this, in formulas (28) and (29) and also in the expressions for stresses and moment stresses variables $x$ and $y$ are replaced, respectively, by values $x-\xi_{j}$ and $y-\eta_{j}$. Then functions $u_{j}\left(x-\xi_{j}, y-\eta_{j}\right), v_{j}\left(x-\xi_{j}, y-\right.$ $\left.\eta_{j}\right), \omega_{j}\left(x-\xi_{j}, y-\eta_{j}\right)$, as well as also functions $\sigma_{x x j}\left(x-\xi_{j}, y-\right.$ $\left.\eta_{j}\right), \sigma_{y y j}\left(x-\xi_{j}, y-\eta_{j}\right), \ldots, \mu_{z y j}\left(x-\xi_{j}, y-\eta_{j}\right)$, have singularities at points $\left(\xi_{j}, \eta_{j}\right)$.

Let us consider the following sums:

$$
\begin{gathered}
\bar{u}=\sum_{j=1}^{N} u_{j}\left(x-\xi_{j}, y-\eta_{j}\right), \\
\bar{v}=\sum_{j=1}^{N} v_{j}\left(x-\xi_{j}, y-\eta_{j}\right), \\
\bar{\omega}=\sum_{j=1}^{N} \omega_{j}\left(x-\xi_{j}, y-\eta_{j}\right), \\
\bar{\sigma}_{x x}=\sum_{j=1}^{N} \sigma_{x x j}\left(x-\xi_{j}, y-\eta_{j}\right), \\
\bar{\sigma}_{y y}=\sum_{j=1}^{N} \sigma_{y y j}\left(x-\xi_{j}, y-\eta_{j}\right), \\
\bar{\mu}_{z y}=\sum_{j=1}^{N} \mu_{z y j}\left(x-\xi_{j}, y-\eta_{j}\right) .
\end{gathered}
$$

For our algorithm approximate solutions of boundary value problems are sought in form (30). Functions (30) satisfy system of (2) everywhere except points $\left(\xi_{j}, \eta_{j}\right), j=1,2, \ldots, N$, where they have a singularity. The unknown coefficients $a_{j}, b_{j}, c_{j}, j=1,2, \ldots, N$, figuring in formulas (28) and (29), 


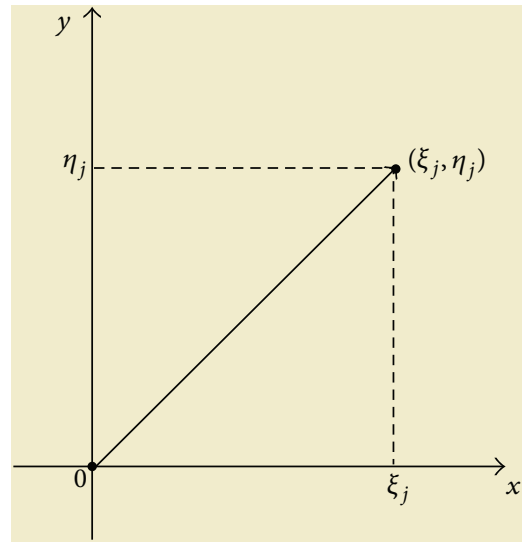

FIGURE 1: Shift of a singular point.

and therefore in formulas (30) too, have to be chosen so that the boundary conditions be satisfied.

For simplicity, let us consider the algorithm of construction of an approximate solution using the example of a boundary value problem for a finite domain with displacements and rotation given on the boundary. Let $\Omega$ be a simply or multiply connected domain with a sufficiently smooth boundary $L$ having almost everywhere the external normal 1. In domain $\Omega$, we seek such a solution of system (2) that satisfies the following boundary conditions on boundary $L$ :

$$
\begin{aligned}
& \left.u_{l}\right|_{L}=f_{1}(x, y), \\
& \left.u_{s}\right|_{L}=f_{2}(x, y), \\
& \left.\omega\right|_{L}=g(x, y),
\end{aligned}
$$

$$
(x, y) \in L,
$$

where $f_{1}, f_{2}$, and $g$ are the functions given on the boundary.

In the first place, on the boundary we more or less uniformly distribute $N$ points with coordinates $\left(x_{1}, y_{1}\right),\left(x_{2}, y_{2}\right), \ldots,\left(x_{N}, y_{N}\right)$. As to points $\left(\xi_{j}, \eta_{j}\right)$, we arrange them on some contour that lies around the external boundary of $\Omega$ and also on the contours within the internal boundaries if the domain is multiply connected as shown in Figure 2.

An approximate solution is sought in the form

$$
\begin{aligned}
\bar{u}_{l} & =\sum_{j=1}^{N} u_{j}\left(x-\xi_{j}, y-\eta_{j}\right) \cos \vartheta \\
& +v_{j}\left(x-\xi_{j}, y-\eta_{j}\right) \sin \vartheta, \\
\bar{u}_{s} & =\sum_{j=1}^{N}-u_{j}\left(x-\xi_{j}, y-\eta_{j}\right) \sin \vartheta \\
& +v_{j}\left(x-\xi_{j}, y-\eta_{j}\right) \cos \vartheta, \\
\bar{\omega} & =\sum_{j=1}^{N} \omega_{j}\left(x-\xi_{j}, y-\eta_{j}\right) .
\end{aligned}
$$

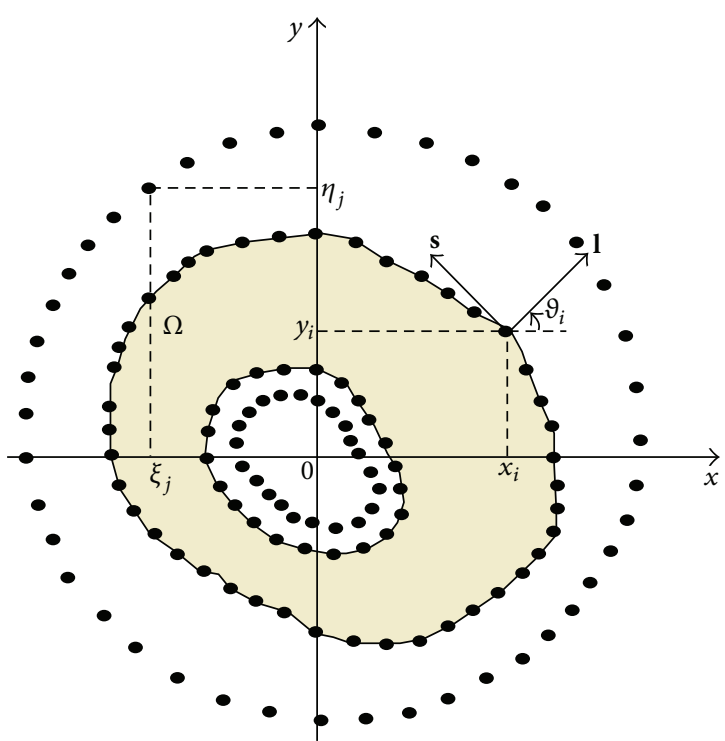

Figure 2: Domain $\Omega$ with singular points $\left(\xi_{j}, \eta_{j}\right)$ lying on some external contour and points $\left(x_{i}, y_{i}\right)$ lying on the boundary contours.

We substitute the coordinates of points $\left(x_{i}, y_{i}\right)$ marked on the boundary and the values of angles $\vartheta_{i}$ in formulas (32) and equate the resulting expressions to the respective values of the boundary functions at these points. As a result we obtain the following system of the equations

$$
\begin{aligned}
& \sum_{j=1}^{N}\left\{u_{j}\left(x_{i}-\xi_{j}, y_{i}-\eta_{j}\right) \cos \vartheta_{i}\right. \\
& \left.\quad+v_{j}\left(x_{i}-\xi_{j}, y_{i}-\eta_{j}\right) \sin \vartheta_{i}\right\}=f_{1}\left(x_{i}, y_{i}\right)
\end{aligned}
$$

$$
\begin{aligned}
& \sum_{j=1}^{N}\left\{-u_{j}\left(x_{i}-\xi_{j}, y_{i}-\eta_{j}\right) \sin \vartheta_{i}\right. \\
& \left.\quad+v_{j}\left(x_{i}-\xi_{j}, y_{i}-\eta_{j}\right) \cos \vartheta_{i}\right\}=f_{2}\left(x_{i}, y_{i}\right),
\end{aligned}
$$

$\sum_{j=1}^{N} \omega_{j}\left(x_{i}-\xi_{j}, y_{i}-\eta_{j}\right)=g\left(x_{i}, y_{i}\right)$,

$$
i=1,2, \ldots, N
$$

But according to formulas (28), the latter system is a system of $3 N$ linear algebraic equations with $3 N$ unknowns $a_{j}, b_{j}, c_{j}, j=1,2, \ldots, N$ :

$$
\begin{aligned}
& \sum_{j=1}^{N}\left\{A_{1 i j} a_{j}+B_{1 i j} b_{j}+C_{1 i j} c_{j}\right\}=f_{1}\left(x_{i}, y_{i}\right), \\
& \sum_{j=1}^{N}\left\{A_{2 i j} a_{j}+B_{2 i j} b_{j}+C_{2 i j} c_{j}\right\}=f_{2}\left(x_{i}, y_{i}\right), \\
& \sum_{j=1}^{N}\left\{A_{3 i j} a_{j}+B_{3 i j} b_{j}+C_{3 i j} c_{j}\right\}=g\left(x_{i}, y_{i}\right),
\end{aligned}
$$


where $i=1,2, \ldots, N$ and $A_{k i j}, B_{k i j}, C_{k i j}, k=1,2,3$, are the already known coefficients. For example, $A_{1 i j}$ has the form

$$
\begin{aligned}
A_{1 i j} & =\frac{1}{2 \mu}\left(\frac{\lambda+3 \mu}{\lambda+\mu} \ln \sqrt{\left(x_{i}-\xi_{j}\right)^{2}+\left(y_{i}-\eta_{j}\right)^{2}}\right. \\
& \left.-\frac{\left(x_{i}-\xi_{j}\right)^{2}}{\left(x_{i}-\xi_{j}\right)^{2}+\left(y_{i}-\eta_{j}\right)^{2}}\right) \cos \vartheta_{i}-\frac{1}{2 \mu} \\
& \cdot \frac{\left(x_{i}-\xi_{j}\right)\left(y_{i}-\eta_{j}\right)}{\left(x_{i}-\xi_{j}\right)^{2}+\left(y_{i}-\eta_{j}\right)^{2}} \sin \vartheta_{i} .
\end{aligned}
$$

After solving system (34), we substitute the obtained coefficient values in the respective expressions (30) and find all the desired displacement and rotation components, as well as all stress and moment stress components.

In an analogous manner we can construct approximate solutions of boundary value problems when stresses and moment stresses are given on the domain boundary. We can also consider all kinds of mixed boundary value problems.

In the algorithm described above we have used Green's functions for the construction of the approximate solution. But, as has already been mentioned in Introduction, the use of Green's functions is not obligatory at all. The harmonic functions and a solution of the Helmholtz equation can be represented as finite series obtained as a result of the separation of variables in a polar system of coordinates but, at the same time, boundary value problems are solved in a Cartesian coordinate system.

Let us give a brief description of the latter approach to the construction of an approximate solution of a boundary value problem. It is assumed for the sake of simplicity that we consider a doubly connected domain $\Omega$ bounded by the simple closed contours $L_{1}, L_{2}$, of which $L_{2}$ encircles $L_{1}$, while $L_{1}$ lies around the origin.

The harmonic functions $\varphi$ and, $\psi$ in the general solutions (19) and (20) are represented as follows:

$$
\begin{aligned}
\varphi= & a \ln r+a_{0}+\sum_{n=1}^{N}\left\{r^{-n}\left[a_{1 n} \cos (n \vartheta)+a_{2 n} \sin (n \vartheta)\right]\right. \\
& \left.+r^{n}\left[a_{3 n} \cos (n \vartheta)+a_{4 n} \sin (n \vartheta)\right]\right\}, \\
\psi & =b \ln r+b_{0}+\sum_{n=1}^{N}\left\{r^{-n}\left[b_{1 n} \cos (n \vartheta)+b_{2 n} \sin (n \vartheta)\right]\right. \\
& \left.+r^{n}\left[b_{3 n} \cos (n \vartheta)+b_{4 n} \sin (n \vartheta)\right]\right\},
\end{aligned}
$$

where $r(x, y)=\sqrt{x^{2}+y^{2}}$ and

$$
\vartheta(x, y)= \begin{cases}\arctan \frac{y}{x}, & x>0, \\ \arctan \frac{y}{x}+\pi, & x<0, y \geq 0, \\ \arctan \frac{y}{x}-\pi, & x<0, y<0, \\ \frac{\pi}{2}, & x=0, y>0, \\ -\frac{\pi}{2}, & x=0, y<0 .\end{cases}
$$

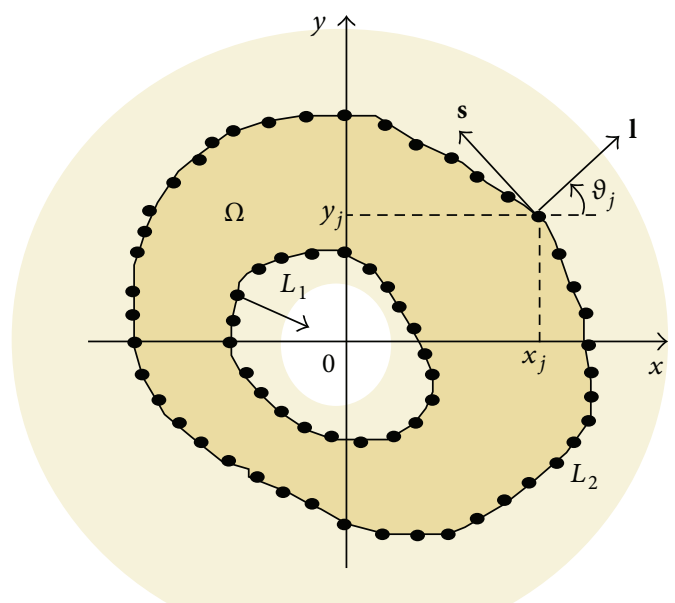

FIGURE 3: The considered doubly connected domain $\Omega$.

Function $\chi$ is written in the form

$$
\begin{aligned}
\chi= & c I_{0}(\gamma r)+c_{0} K_{0}(\gamma r) \\
& +\sum_{n=1}^{N}\left\{I_{n}(\gamma r)\left[c_{1 n} \cos (n \vartheta)+c_{2 n} \sin (n \vartheta)\right]\right. \\
& \left.+K_{n}(\gamma r)\left[c_{3 n} \cos (n \vartheta)+c_{4 n} \sin (n \vartheta)\right]\right\},
\end{aligned}
$$

where $I_{n}(\gamma r)$ and $K_{n}(\gamma r)$ are, respectively, a modified Bessel and a Macdonald function of order $n$ [45].

Substituting formulas (36) and (38) in the general solutions (19) and (20), we obtain the analytical type of the desired approximate solution. However this solution contains the unknown coefficients $a, a_{0}, b, b_{0}, c, c_{0}, a_{\alpha n}, b_{\alpha n}, c_{\alpha n}, n=$ $1,2, \ldots, N, \alpha=1,2,3,4$.

Further, we more or less uniformly distribute $2(2 \mathrm{~N}+$ $1)$ points with coordinates $\left(x_{1}, y_{1}\right),\left(x_{2}, y_{2}\right), \ldots,\left(x_{2(2 N+1)}\right.$, $\left.y_{2(2 N+1)}\right)$ on contours $L_{1}$ and $L_{2}$ (Figure 3 ). In order that the given boundary conditions be satisfied at these points (Figure 3), we substitute the coordinates of the boundary points $\left(x_{i}, y_{i}\right), i=1,2, \ldots, 2(2 N+1)$, in the respective formulas of the approximate solution and equate the resulting expressions to the known values of the boundary conditions at these points. Thus, for coefficients $a, a_{0}, b, b_{0}, c, c_{0}, a_{\alpha n}, b_{\alpha n}, c_{\alpha n}, n=1,2, \ldots, N, \alpha=1,2,3,4$, we obtain a system of $6(2 N+1)$ linear algebraic equations with $6(2 N+1)$ unknowns. By solving this system we define the values of all the unknown coefficients in the formulas for an approximate solution and thereby accomplish the construction of the approximate solution.

In Section 5, by the above technique not requiring the use of Green's functions we solve a nonlocal problem of the Bitsadze-Samarskii type [46-49]. We want to show by the above example that the proposed method can be used for constructing approximate solutions not only of classical boundary value problems but also of some nonclassical ones.

As to the accuracy of approximate solutions constructed by the proposed method, we can say the following. Since 
in our case an approximate solution of a boundary value problem has the analytical form, we can easily calculate the values of this solution on the domain boundary. If the result obtained is sufficiently close to the given boundary conditions, then provided that the problem is well posed, we can conclude that the constructed solution is sufficiently close to an exact solution since the equilibrium equations are satisfied exactly within the domain.

\section{Examples}

We take the following elastic constants of the Cosserat medium: $\lambda=82.098765432 \mathrm{GPa}, \mu=35.185185185 \mathrm{GPa}$, $\alpha=7.037037036 \mathrm{GPa}$, and $\nu+\beta=0.7037037036 \mathrm{GN}$. These data correspond to the elastic characteristics of brass.

Example 1. Let domain $\Omega_{1}$ be a square with a circular hole $\Omega_{1}=\Omega_{10} \backslash \bar{\Omega}_{0}$, where $\Omega_{10}=\{(x, y) \mid-3<x<3,-3<y<3\}$ and $\Omega_{0}=\left\{(x, y) \mid x^{2}+y^{2}<4\right\}$ (Figure 4).

Consider the so-called Kirsch problem [19, 45] for domain $\Omega_{1}$ when the constant normal stresses are applied to the two opposite sides of the square, and the remaining part of the boundary is free from stresses (Figure 5). Thus we solve the following boundary value problem:

$$
\begin{aligned}
\sigma_{x x} & =1.0, \\
\sigma_{x y} & =0, \\
\mu_{x z} & =0, \\
& x= \pm 3,-3<y<3, \\
\sigma_{y y} & =0, \\
\sigma_{y x} & =0, \\
\mu_{y z} & =0, \\
\sigma_{l l} & =0, \\
\sigma_{l s} & =0, \\
\mu_{l z} & =0,
\end{aligned}
$$

This problem is solved approximately by the proposed method both for the Cosserat medium and for the classical elastic medium.

We arrange points $\left(\xi_{j}, \eta_{j}\right), j=1,2, \ldots, 72$, on the square containing domain $\Omega_{1}$ (48 points) and on the circumference within the internal contour (24 points) as is shown in Figure 6. On the domain boundary we also mark 72 points at which the given boundary conditions are satisfied. In the general case, for the unknown coefficients we obtain $72 \times$ $3=216$ linear algebraic equations with the same number of unknowns. If the problem is symmetric with respect to the coordinate axes, then the number of unknown coefficients and the number of equations can be reduced. In that case, in

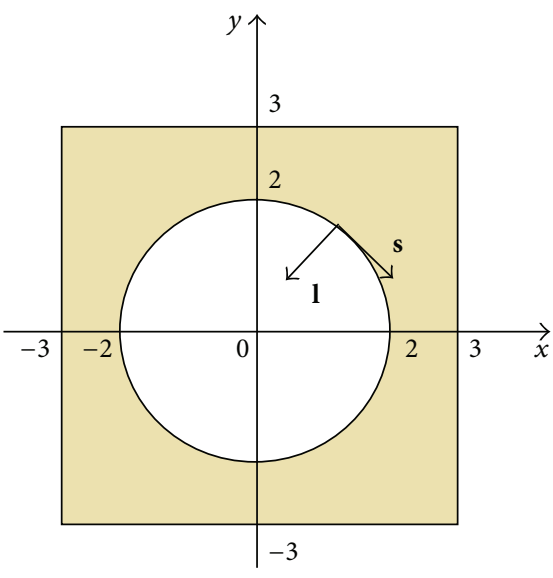

FIgURE 4: The considered domain $\Omega_{1}$.

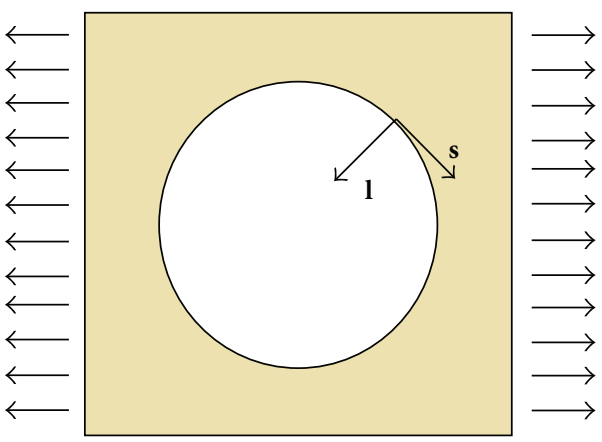

FIGURE 5: Unilateral tension of domain $\Omega_{1}$.

the equations we preserve only the coefficients corresponding to the first quadrant and express the remaining coefficients through them according to the formulas

$$
\begin{aligned}
& a_{j}=-a_{25-j}, \\
& b_{j}=b_{25-j}, \\
& c_{j}=-c_{25-j}, \\
& a_{j}=-a_{j-24}, \\
& b_{j}=-b_{j-24}, \\
& c_{j}=c_{j-24}, \quad j=25,26, \ldots, 36, \ldots, 24, \\
& a_{j}=a_{49-j}, \\
& b_{j}=-b_{49-j}, \\
& c_{j}=-c_{49-j}, \quad
\end{aligned}
$$




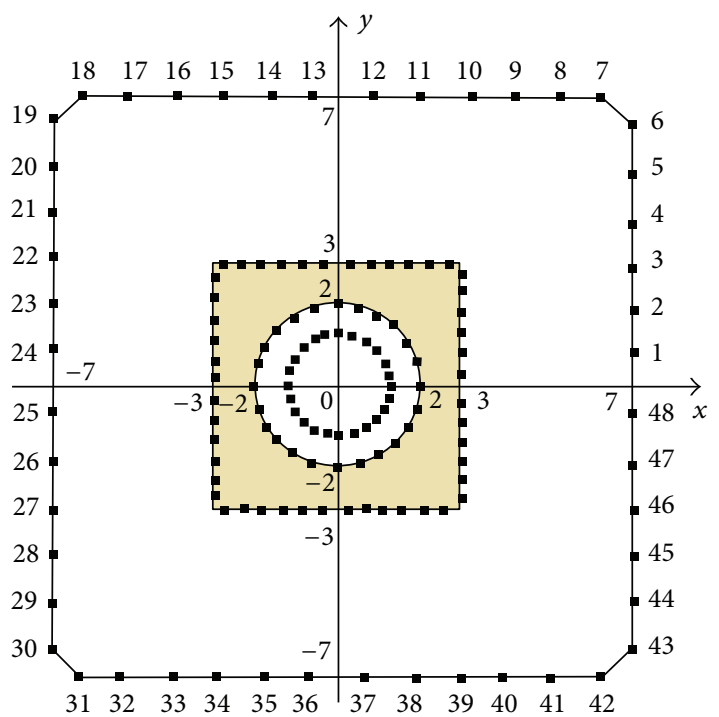

FIGURE 6: Domain $\Omega_{1}$ encircled by singular points.

$$
\begin{aligned}
& a_{j}=-a_{110-j}, \\
& b_{j}=b_{110-j}, \\
& c_{j}=-c_{110-j}, \\
& a_{j}=-a_{j-12}, \\
& b_{j}=-b_{j-12}, \\
& c_{j}=c_{j-12}, \quad j=66,57, \ldots, 61, \\
& a_{j}=a_{122-j}, \\
& b_{j}=-b_{122-j}, \\
& c_{j}=-c_{122-j},
\end{aligned}
$$

After solving the resulting system we compared the boundary values of the obtained solutions with the given boundary conditions. If absolute errors of order $10^{-2}$ are regarded as small from the practical point of view (errors are especially noticeable near the angular points), then the resulting solution can be regarded as satisfactory. If a higher exactness is needed, it can be achieved by increasing the number of points on the boundary where the boundary conditions are satisfied or by a more appropriate choice of the contour on which the singular points are arranged. For some problems admitting an exact solution, we have managed to construct the approximate solutions with absolute errors of order not higher than $10^{-6}$.

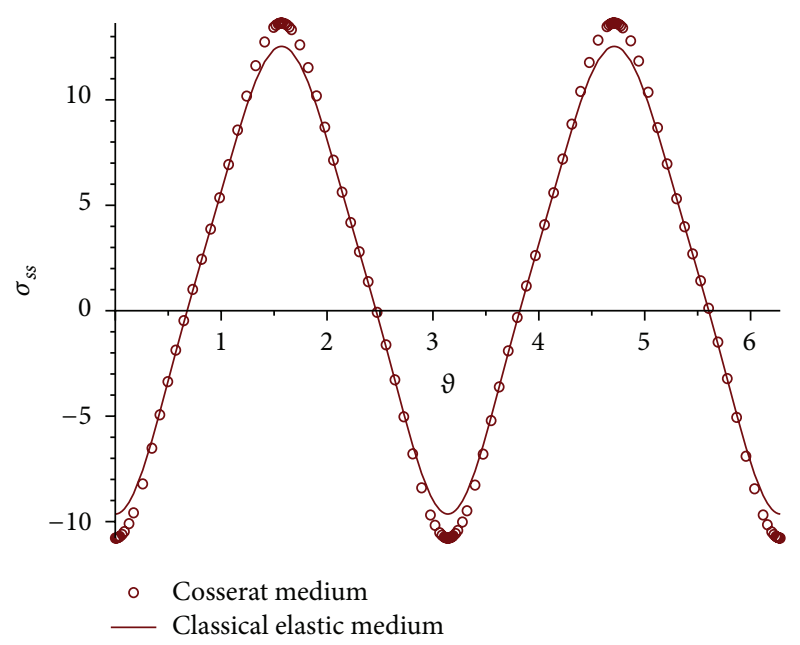

FIGURE 7: Graphs of the distribution of stress $\sigma_{s s}$ along the hole contour for the classical elastic medium and the Cosserat medium for domain $\Omega_{1}$.

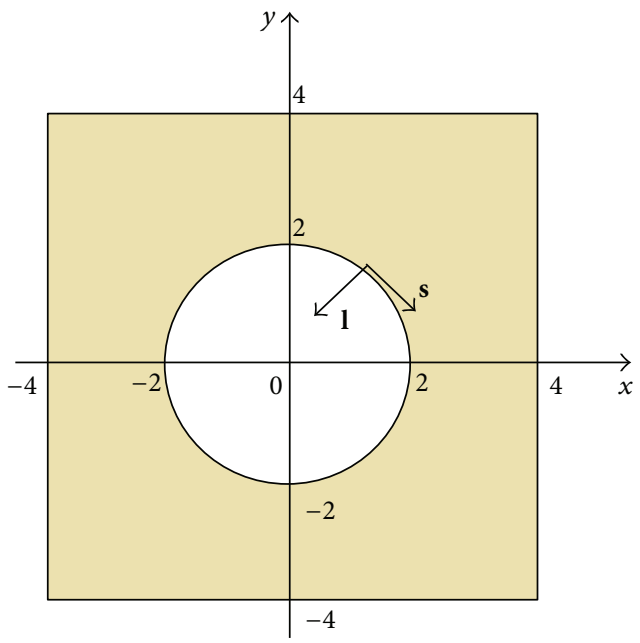

FIgURE 8: The considered domain $\Omega_{2}$.

In the case of the Kirsch problem it is of special interest to investigate the distribution of stress $\sigma_{s s}$ along the hole contour. A maximal value of stress $\sigma_{s s}$ both for the classical elastic medium and for the Cosserat medium is obtained, as expected, at $\vartheta= \pm \pi / 2$. For the classical medium, $\left(\sigma_{s s}\right)_{\max }=$ 13.7 and this value does not depend on the material (if the material is isotropic and homogenous). For the Cosserat medium, $\left(\sigma_{s s}\right)_{\max }$ depends on the material and in our case its value is $\left(\sigma_{s s}\right)_{\max }=12.5$ (Figure 7 ).

Example 2. Now let us consider the Kirsch problem for domain $\Omega_{2}=\Omega_{20} \backslash \bar{\Omega}_{0}, \Omega_{20}=\{(x, y) \mid-4<x<4,-4<y<$ 4\} (Figure 8).

As expected, in this case the values of $\left(\sigma_{s s}\right)_{\max }$ diminish both for the classical medium and for the Cosserat medium and the difference between them becomes even more insignificant (see Figure 9). We know from the literature that in the case of an infinite domain with a circular 


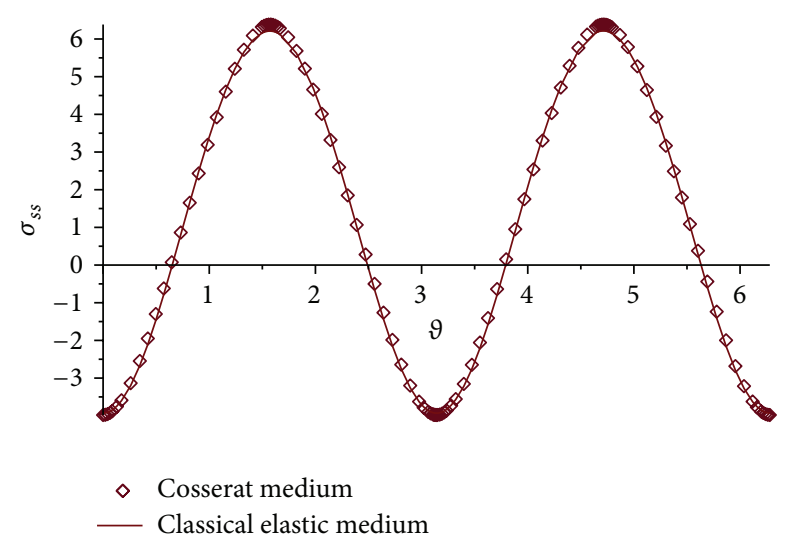

FIGURE 9: Graphs of the distribution of stress $\sigma_{s s}$ along the hole contour for the classical medium and the Cosserat medium for domain $\Omega_{2}$.

hole value $\left(\sigma_{s s}\right)_{\max }$ for the Cosserat medium is smaller than the stress concentration coefficient for the classical elastic medium [20] which is equal to three. Therefore, as the ratio of $a / r$, where $a$ is the side of the square and $r$ is the hole radius, increases to a certain value, values $\left(\sigma_{s s}\right)_{\max }$ for the classical medium and for the Cosserat medium get closer to each other.

Finally, let us we consider the nonlocal problem for a rectangular domain for the classical elastic medium.

Example 3. We consider domain $V=\{-2.5<x<2.5,-2<$ $y<2\}$ (Figure 10). In domain $V$, it is required to find a solution of system (2) (where $\lambda=2, \mu=1, \alpha=0$, and $\nu+\beta=$ $0)$ that satisfies the following conditions:

$$
\begin{gathered}
u=-0.38 y^{2} \pm 1.5 y+0.125, \\
x= \pm 2.5,-2 \leq y \leq 2, \\
v=-0.14 y^{2} \pm 0.5 y-2.125, \\
x= \pm 2.5,-2 \leq y \leq 2, \\
\left.\sigma_{y y}\right|_{y=2}-\left.\sigma_{y y}\right|_{y=1}=0.08, \quad-2.5<x<2.5, \\
\left.\sigma_{y x}\right|_{y=2}=-0.08 x-1.12, \quad-2.5<x<2.5, \\
u=0.02 x^{2}-1.2 x-1.52, \\
y=-2,-2.5<x<2.5, \\
v=-0.34 x^{2}-0.4 x-0.56, \\
y=-2,-2.5<x<2.5 .
\end{gathered}
$$

The exact solution of this problem is as follows:

$$
\begin{aligned}
& u=0.02 x^{2}-0.38 y^{2}+0.6 x y, \\
& v=-0.34 x^{2}-0.14 y^{2}+0.2 x y .
\end{aligned}
$$

The boundary contour of the considered domain is divided by points into 72 equal segments. 19 points are also

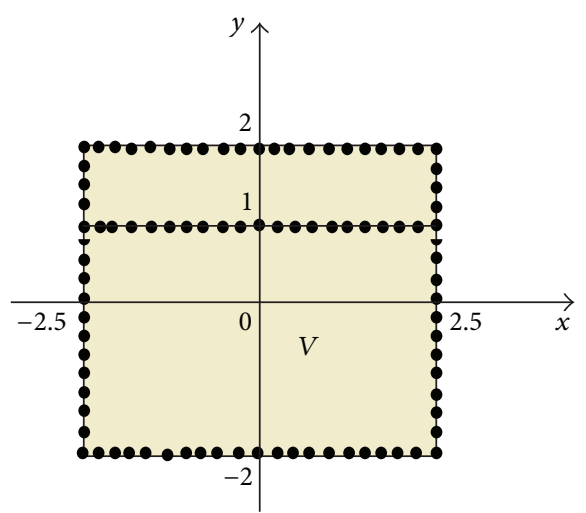

FIGURE 10: Domain $V$ in which the nonlocal problem of plane elasticity is solved.

distributed uniformly on a segment within the domain with given nonlocal conditions. An approximate solution is sought in the form

$$
\begin{aligned}
u= & 0.5 \sum_{n=1}^{36} r^{n-1}\left\{\left[\frac{5}{6} r \cos (n 9)-\frac{n}{2} y \sin ((n-1) \vartheta)\right.\right. \\
& \left.-\frac{n}{2} x \cos ((n-1) \vartheta)\right] a_{n}+\left[\frac{5}{6} r \sin (n \vartheta)\right. \\
& \left.+\frac{n}{2} y \cos ((n-1) \vartheta)-\frac{n}{2} x \sin ((n-1) \vartheta)\right] b_{n}-n \\
& \left.\cdot \sin ((n-1) \vartheta) c_{n}+n \cos ((n-1) \vartheta) d_{n}\right\}, \\
v= & 0.5 \sum_{n=1}^{36} r^{n-1}\left\{\left[\frac{5}{6} r \sin (n \vartheta)+\frac{n}{2} x \sin ((n-1) \vartheta)\right.\right. \\
& \left.-\frac{n}{2} x \cos ((n-1) \vartheta)\right] a_{n}-\left[\frac{5}{6} r \cos (n \vartheta)\right. \\
& \left.+\frac{n}{2} y \cos ((n-1) \vartheta)+\frac{n}{2} x \sin ((n-1) \vartheta)\right] b_{n}-n \\
& \left.\cdot \cos ((n-1) \vartheta) c_{n}-n \sin ((n-1) \vartheta) d_{n}\right\} .
\end{aligned}
$$

The stress tensor components $\sigma_{y y}$ and $\sigma_{y x}$ figuring in the initial condition are represented as finite series:

$$
\begin{aligned}
\sigma_{y y} & =\sum_{n=1}^{36} n r^{n-2}\{[r \cos ((n-1) \vartheta) \\
& \left.+\frac{n-1}{2} y \sin ((n-2) \vartheta)+\frac{n-1}{2} x \cos ((n-2) \vartheta)\right] \\
& \cdot a_{n}+\left[r \sin ((n-1) \vartheta)-\frac{n-1}{2} y \cos ((n-2) \vartheta)\right. \\
& \left.+\frac{n-1}{2} x \sin ((n-2) \vartheta)\right] b_{n}+(n-1) \sin ((n-2) \vartheta) \\
& \left.\cdot c_{n}-(n-1) \cos ((n-2) \vartheta) d_{n}\right\},
\end{aligned}
$$


TABLE 1: Numerical results for Example 3.

\begin{tabular}{lccc}
\hline \multirow{2}{*}{$(x, y)$} & $\bar{u}(x, y)$ & $u(x, y)$ & $|\bar{u}-u|$ \\
& $\bar{v}(x, y)$ & $v(x, y)$ & $|\bar{v}-v|$ \\
\hline \multirow{2}{*}{$(0,0)$} & $-7.205904960 \cdot 10^{-9}$ & 0 & $7.2059 \cdot 10^{-9}$ \\
& $-2.170915985 \cdot 10^{-7}$ & 0 & $2.171 \cdot 10^{-7}$ \\
\hline \multirow{2}{*}{$(1.0,1.0)$} & 0.2399999816 & 0.24 & $1.84 \cdot 10^{-8}$ \\
& -0.2800003047 & -0.28 & $3.047 \cdot 10^{-7}$ \\
\hline \multirow{2}{*}{$(-1.5,1.5)$} & -2.159999943 & -2.16 & $5.7 \cdot 10^{-8}$ \\
& -1.530000297 & -1.53 & $2.97 \cdot 10^{-7}$ \\
\hline \multirow{2}{*}{$(1.2,1.3)$} & 0.3225999578 & 0.3226 & $4.22 \cdot 10^{-8}$ \\
& -0.4142003246 & -0.4142 & $3.346 \cdot 10^{-7}$ \\
\hline \multirow{2}{*}{$(-1.8,-2.3)$} & 0.5386005059 & 0.5386 & $5.059 \cdot 10^{-7}$ \\
& -1.014199600 & -1.0142 & $4 \cdot 10^{-7}$ \\
\hline \multirow{2}{*}{$(2.2,-1.4)$} & -2.496000007 & -2.4960 & $7 \cdot 10^{-9}$ \\
& -2.535999981 & -2.5360 & $1.9 \cdot 10^{-8}$ \\
\hline \multirow{2}{*}{$(1.25,2.15)$} & -0.1128001215 & -0.1128 & $1.215 \cdot 10^{-7}$ \\
& -0.6409003984 & -0.64090 & $3.984 \cdot 10^{-7}$ \\
\hline
\end{tabular}

$$
\begin{aligned}
\sigma_{y x} & =\sum_{n=1}^{36} \frac{n(n-1)}{2} r^{n-2}\{[-y \cos ((n-2) \vartheta) \\
& +x \sin ((n-2) \vartheta)] a_{n}-[y \sin ((n-2) \vartheta) \\
& +x \cos ((n-2) \vartheta)] b_{n}-2 \cos ((n-2) \vartheta) c_{n}-2 \\
& \left.\cdot \sin ((n-2) \vartheta) d_{n}\right\} .
\end{aligned}
$$

Next we substitute the coordinates of the points marked on the boundary and within the domain into the last four formulas and thus satisfy at these points the corresponding boundary and nonlocal conditions. As a result we obtain the system consisting of 144 linear algebraic equations and containing 144 unknowns $\left(a_{1}, \ldots, a_{36}, b_{1}, \ldots, b_{36}, c_{1}, \ldots, c_{36}, d_{1}, \ldots, d_{36}\right)$. After solving this system by the above formulas we can easily define the displacement vector and stress tensor components.

In our study we used Maple 17 software. The numerical results are presented in Table 1 , where $\bar{u}$ and $\bar{v}$ denote the approximate values of the displacement vector components.

From the numerical results we see that the proposed technique gives the good approximate solution for the considered nonlocal mixed boundary value problem of plane elasticity.

\section{Conclusion}

In this paper, we consider the static case of plane deformation for the elastic Cosserat medium. The constructed general solution of the corresponding system of differential equations is represented by two harmonic functions and a solution of the Helmholtz equation. Based on this solution, we propose the algorithm for the approximate solution of a wide class of boundary value problems.

The same boundary value problems can be solved by the method of Green's functions or boundary elements. Our technique, based on representing the solution through two harmonic functions and a solution of the Helmholtz equation, is an alternative method, different from them.

To show the validity of the proposed algorithm for moment elasticity, we construct the approximate solutions of boundary value problems of stress concentration in a rectangular domain with circular holes. The stress concentration coefficients are calculated along the hole contour and their values are compared with those for the classical elastic case.

For the classical elastic medium we also obtain the approximate solution of a nonlocal problem and compare it with the known exact solution.

We believe that the proposed algorithm can be applied for constructing an approximate solution of a wide class of boundary value problems for systems of partial differential equations whose general solutions can be represented by means of the solutions of relatively simple equations of mathematical physics.

\section{Conflict of Interests}

The author declares that there is no conflict of interests regarding the publication of this paper.

\section{References}

[1] E. Cosserat and F. Cosserat, Theorie des Corps Deformables, Hermann, Paris, France, 1909.

[2] C. Truesdell and R. A. Toupin, "The classical field theories," in Handbuch der Physik, Bd. III/1, S. Flügge, Ed., Springer, Berlin, Germany, 1960.

[3] G. Grioli, "Elasticità assimetrica," Annali di Matematica Pura ed Applicata, Serie IV, vol. 50, pp. 389-417, 1960.

[4] E. Kuvshinskii and E. L. Aero, "Continuum theory of asymmetric elasticity. Equilibrium of isotropic body," Fizika Tverdogo Tela, vol. 6, no. 9, pp. 2689-2699, 1964 (Russian).

[5] R. D. Mindlin, "Influence of couple-stress on stress concentrations," Experimental Mechanics, vol. 3, no. 1, pp. 1-7, 1963.

[6] V. A. Pal'mov, "Fundamental equations of the theory of asymmetric elasticity," Journal of Applied Mathematics and Mechanics, vol. 28, pp. 496-505, 1964 (Russian).

[7] A. C. Eringen and E. S. Suhubi, "Nonlinear theory of simple micro-elastic solids-I," International Journal of Engineering Science, vol. 2, no. 2, pp. 189-203, 1964.

[8] W. Novacki, "Couple stresses in the theory of thermoelasticity," Bulletin of the Polish Academy of Sciences: Technical Sciences, vol. 14 , no. 8,1966 .

[9] A. E. Green and P. M. Naghdi, "The linear theory of an elastic Cosserat plate," Mathematical Proceedings of the Cambridge Philosophical Society, vol. 63, no. 2, pp. 537-550, 1967.

[10] W. Novacki, "On the completeness of stress functions in asymmetric elasticity," Bulletin of the Polish Academy of Sciences: Technical Sciences, vol. 14, no. 7, 1968.

[11] V. D. Kupradze, T. G. Gegelia, M. O. Basheleishvili, and T. V. Burchuladze, Three-Dimensional Problems of the Mathematical Theory of Elasticity and Thermoelasticity, North-Holland, Amsterdam, Netherlands; Nauka, Moscow, Russia, 1979.

[12] W. Novacki, Theory of Asymmetric Elasticity, Polish Scientific, Warsaw, Poland, 1986.

[13] D. Natroshvili, R. Gachechiladze, A. Gachechiladze, and I. G. Stratis, "Transmission problems in the theory of elastic 
hemitropic materials," Applicable Analysis, vol. 86, no. 12, pp. 1463-1508, 2007.

[14] D. Natroshvili, L. Giorgashvili, and I. G. Stratis, "Mathematical problems of the theory of elasticity of chiral materials," Applied Mathematics, Informatics and Mechanics, vol. 8, no. 1, pp. 47103, 127, 2003.

[15] R. Janjgava and B. Gulua, "About plane theory for hemitropic elastic materials," Reports of Enlarged Session of the Seminar of VIAM, vol. 19, no. 1, pp. 38-42, 2004.

[16] T. V. Meunargia, Development of a Method of I. N. Vekua for Problems of the Three-Dimensional Moment Theory of Elasticity, TSU Publishing, Tbilisi, Georgia, 1987 (Russian).

[17] P. Schiavone, "Fundamental sequences of functions in the approximation of solutions to exterior problems in the bending of thin micropolar plates," Applicable Analysis, vol. 35, no. 1-4, pp. 263-274, 1990.

[18] J. D. Kaplunov and H. Lippmann, "Elastic-plastic torsion of a Cosserat-type rod," Acta Mechanica, vol. 113, no. 1-4, pp. 53-62, 1995.

[19] É. A. Bulanov, "On the moment theory of elasticity. Plane deformation. Part 3," Strength of Materials, vol. 30, no. 5, pp. 516-521, 1998.

[20] M. A. Kulesh, V. P. Matveenko, and I. N. Shardakov, "Construction and analysis of exact analytical solution of kirsch problem within the cosserat continuum and pseudo-continuum," Journal of Applied Mechanics and Technical Physics, vol. 42, no. 4, pp. 145-154, 2001 (Russian).

[21] E. Providas and M. A. Kattis, "Finite element method in plane Cosserat elasticity," Computers \& Structures, vol. 80, no. 27-30, pp. 2059-2069, 2002.

[22] M. A. Kulesh, V. P. Matveenko, and I. N. Shardakov, "Parametric analysis of analytical solutions to one- and twodimensional problems in couple-stress theory of elasticity," ZAMM: Zeitschrift für Angewandte Mathematik und Mechanik, vol. 83, no. 4, pp. 238-248, 2003.

[23] A. Zeghadi, S. Forest, A. Gourgues, and O. Bouaziz, "Cosserat continuum modelling of grain size effects in metal polycrystals," PAMM, vol. 5, no. 1, pp. 79-82, 2005.

[24] H. W. Zhang, H. Wang, and G. Z. Liu, "Quadrilateral isoparametric finite elements for plane elastic Cosserat bodies," Acta Mechanica Sinica, vol. 21, no. 4, pp. 388-394, 2005.

[25] S. Potapenko, "A generalized fourier approximation in antiplane Cosserat elasticity," Journal of Elasticity, vol. 81, no. 2, pp. 159-177, 2005.

[26] M. Bîrsan, "On Saint-Venant's principle in the theory of Cosserat elastic shells," International Journal of Engineering Science, vol. 45, no. 2-8, pp. 187-198, 2007.

[27] M. Bîrsan, "Inequalities of Korn's type and existence results in the theory of Cosserat elastic shells," Journal of Elasticity, vol. 90, no. 3, pp. 227-239, 2008.

[28] S. O. Sarkisyan, "Boundary value problems of the asymmetric theory of elasticity for thin plates," Journal of Applied Mathematics and Mechanics, vol. 72, no. 1, pp. 129-147, 2008.

[29] J. Jeong, H. Ramezani, I. Munch, and P. Neff, "A numerical study for linear isotropic Cosserat elasticity with conformally invariant curvature," Zeitschrift für Angewandte Mathematik und Mechanik, vol. 89, no. 7, pp. 552-569, 2009.

[30] H. Altenbach and V. A. Eremeyev, "On the linear theory of micropolar plates," Zeitschrift für Angewandte Mathematik und Mechanik, vol. 89, no. 4, pp. 242-256, 2009.
[31] A. Riahi and J. H. Curran, "Full 3D finite element Cosserat formulation with application in layered structures," Applied Mathematical Modelling, vol. 33, no. 8, pp. 3450-3464, 2009.

[32] J. Altenbach, H. Altenbach, and V. A. Eremeyev, "On generalized Cosserat-type theories of plates and shells: a short review and bibliography," Archive of Applied Mechanics, vol. 80, no. 1, pp. 73-92, 2010.

[33] E. F. Grekova, "Linear reduced cosserat medium with spherical tensor of inertia, where rotations are not observed in experiment," Mechanics of Solids, vol. 47, no. 5, pp. 538-543, 2012.

[34] V. V. Korepanov, V. P. Matveenko, and I. N. Shardakov, "Finite element analysis of two- and three-dimensional static problems in the asymmetric theory of elasticity as a basis for the design of experiments," Acta Mechanica, vol. 223, no. 8, pp. 1739-1750, 2012.

[35] S. De Cicco and D. Ieşan, "A theory of chiral Cosserat elastic plates," Journal of Elasticity, vol. 111, no. 2, pp. 245-263, 2013.

[36] P. Neff, M. Bîrsan, and F. Osterbrink, "Existence theorem for geometrically nonlinear Cosserat micropolar model under uniform convexity requirements," Journal of Elasticity, vol. 121, no. 1, pp. 119-141, 2015.

[37] V. D. Kupradze and M. A. Aleksidze, “The method of functional equations for the approximate solution of certain boundary value problems," USSR Computational Mathematics and Mathematical Physics, vol. 4, no. 4, pp. 82-126, 1964.

[38] M. A. Aleksidze, Solution of Boundary Value Problems by Expansion in Non-Orthogonal Functions, Nauka, Moscow, Russia, 1978 (Russian).

[39] M. A. Aleksidze, Fundamental Functions in Approximate Solutions of Boundary Value Problems, Nauka, Moscow, Russia, 1991 (Russian).

[40] S. L. Crouch and A. M. Starfield, Boundary Element Methods in Solid Mechanics, George Allen \& Unwin, London, UK, 1983.

[41] C. A. Brebbia, J. C. F. Telles, and L. C. Wrobel, Boundary Element Teqhniques. Theory and Applications in Engineering, Springer, New York, NY, USA, 1984.

[42] P. K. Banerjee, The Boundary Element Methods in Engineering, McGraw-Hill College, London, UK, 1994.

[43] G. Beer, I. Smith, and C. Duenser, The Boundary Element Method with Programming: For Engineers and Scientists, Springer, Vienna, Austria, 2008.

[44] J. T. Katsikadelis, Boundary Elements: Theory and Applications, Elsevier science, Oxford, UK, 2002.

[45] N. N. Lebedev, Special Functions and Their Applications, Prentice-Hall, 1965.

[46] A. V. Bitsadze and A. A. Samarskii, "On some simple generalizations of linear elliptic problems," Doklady Akademii Nauk SSSR, vol. 185, no. 4, pp. 739-740, 1969 (Russian).

[47] A. V. Bitsadze, "On the theory of nonlocal boundary value problems," Doklady Akademii Nauk SSSR, vol. 277, no. 1, pp. 1719, 1984 (Russian).

[48] D. G. Gordeziani, "On one method for solving the boundary value problem of Bitsadze-Samarskii," Seminar of Institute of Applied Mathematics of Tbilisi State University, Annotation of Reports, vol. 2, pp. 39-40, 1970 (Russian).

[49] D. G. Gordeziani, On Methods for Solving a Class of Nonlocal Boundary Value Problems, TSU Press, Tbilisi, Georgia, 1981 (Russian). 


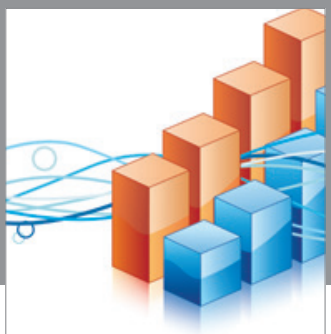

Advances in

Operations Research

vatem alat4

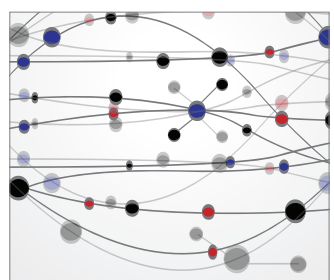

\section{The Scientific} World Journal
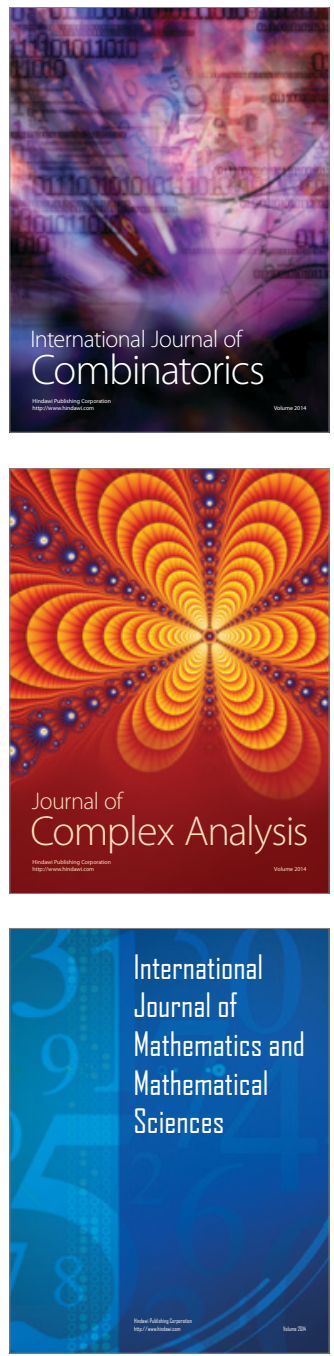
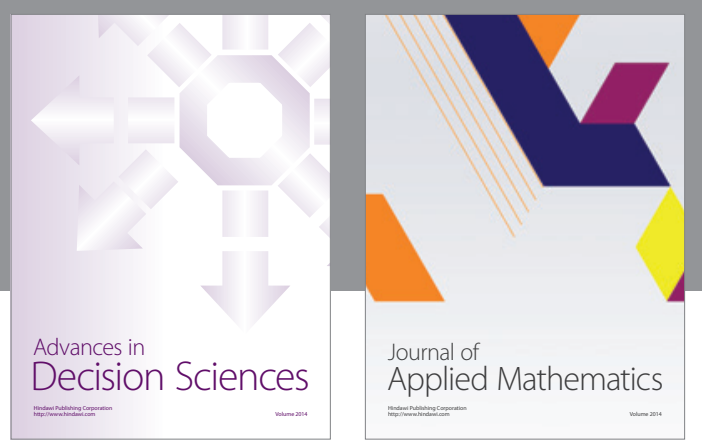

Algebra

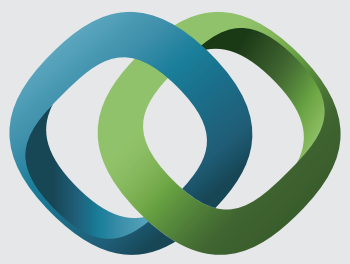

\section{Hindawi}

Submit your manuscripts at

http://www.hindawi.com
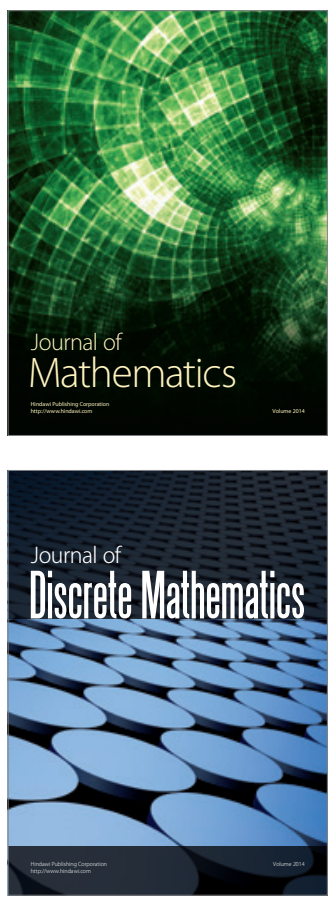

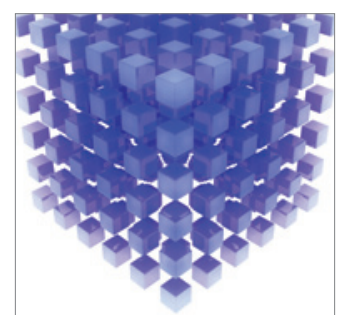

Mathematical Problems in Engineering
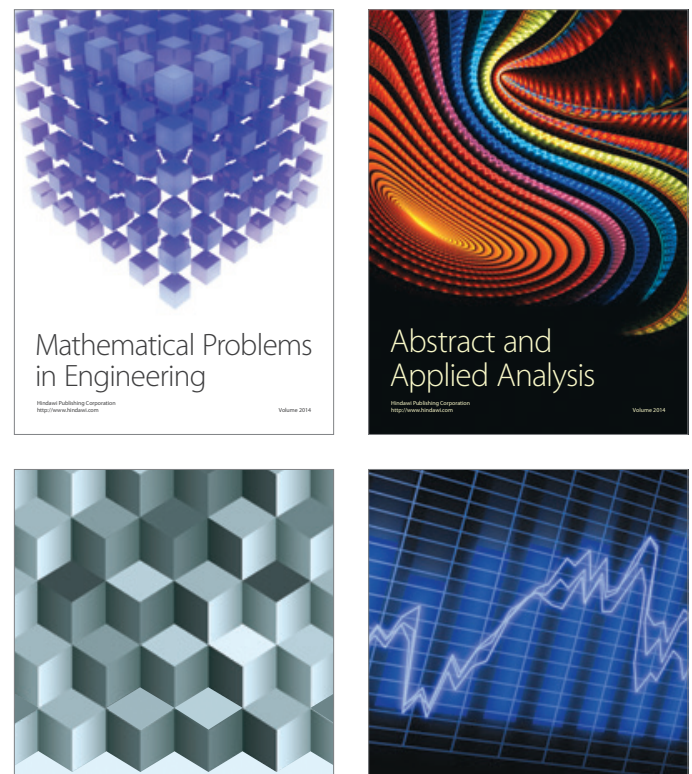

Journal of

Function Spaces

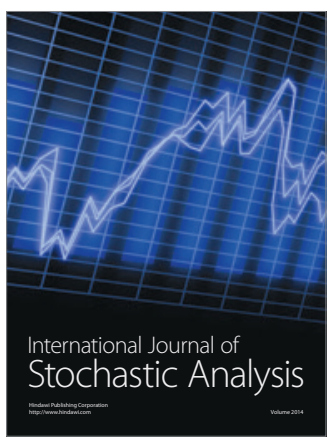

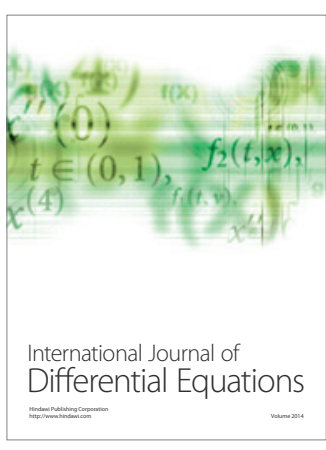
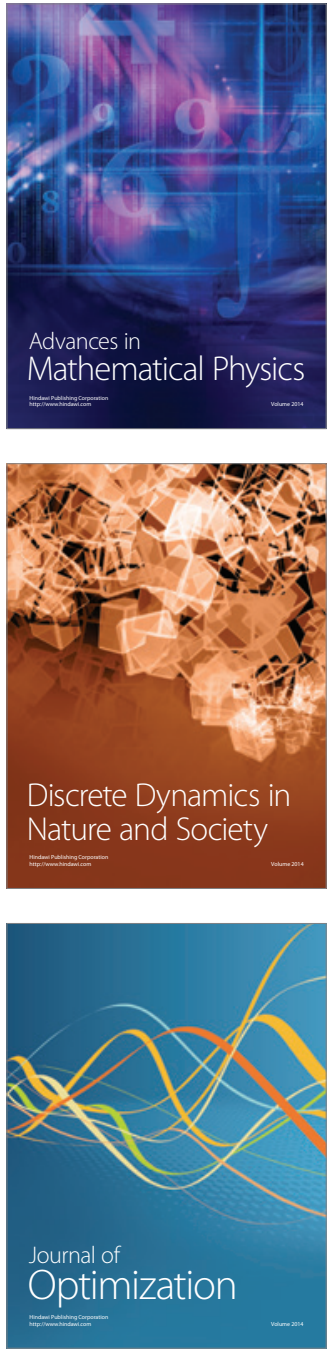